\title{
Neonatal brain and body imaging in the MR-compatible incubator
}

\author{
Monika Bekiesińska-Figatowska ${ }^{1, A-F}$, Magdalena Rutkowska ${ }^{2, B, D}$, Joanna Stankiewicz 3 3,B,D, Katarzyna Krupa ${ }^{1, B-D}$, \\ Beata Iwanowska ${ }^{1, B, C}$, Anna Romaniuk-Doroszewska ${ }^{1, B, C}$, Sylwia Szkudlińska-Pawlak ${ }^{1, B, C}$, Agnieszka Duczkowska1,B,C, \\ Marek Duczkowski1 ${ }^{1, B, C}$, Hanna Brągoszewska ${ }^{1, B, C}$, Jarosław Mądzik ${ }^{1, B, C}$, Piotr Kwaśniewic ${ }^{1, B, C}$, Astra Cabaj ${ }^{1, B, C}$, Ewa Helwich ${ }^{2, E, F}$ \\ ${ }^{1}$ Department of Diagnostic Imaging, Institute of Mother and Child, Warszawa, Poland \\ ${ }^{2}$ Clinic of Neonatology and Neonatal Intensive Care, Institute of Mother and Child, Warszawa, Poland \\ ${ }^{3}$ Clinic of Anesthesiology and Intensive Care, Institute of Mother and Child, Warszawa, Poland \\ A - research concept and design; B - collection and/or assembly of data; C - data analysis and interpretation; \\ $D$ - writing the article; $E$ - critical revision of the article; $F$ - final approval of the article
}

Address for correspondence

Monika Bekiesińska-Figatowska

E-mail:zaklad.rtg@imid.med.pl

\section{Funding sources}

Preparation of this manuscript was supported by an internal grant of the Institute of Mother and Child, Warszawa, Poland (No. 510-14-03).

Conflict of interest

None declared

Received on January 12, 2018

Reviewed on March 22, 2018

Accepted on August 9, 2018

Published online on May 20, 2019

Cite as

Bekiesińska-Figatowska M, Rutkowska M, Stankiewicz J. Neonatal brain and body imaging in the MR-compatible incubator. Adv Clin Exp Med. 2019;28(7):945-954. doi:10.17219/acem94155

DOI

10.17219/acem/94155

Copyright

Copyright by Author(s)

This is an article distributed under the terms of the

Creative Commons Attribution Non-Commercial License

(http://creativecommons.org/licenses/by-nc-nd/4.0/)

\section{Abstract}

Background. The use of a specialized MR-compatible incubator (INC) is very poorly described in the literature and only with regard to brain imaging.

Objectives. To present our own experience with brain and body magnetic resonance imaging (MRI) in the INC in a large cohort of neonates.

Material and methods. A total of 555 examinations were performed in 530 newborns with the use of a 1.5T system and Nomag IC 1.5 incubator, equipped with head and body coils.

Results. More than half of neonates (54\%) were prematurely born at $22+6-36+6$ gestational weeks. They were examined from the first to 153 days of life (median: 18.5, mean: 37.7) with body weights $600-5000 \mathrm{~g}$ (mean: $3051 \mathrm{~g}$ ), $23 \%$ of less than $2500 \mathrm{~g}$. The proportion of brain MRls to other body regions was 533:85 = 86\%:14\%. In 36.6\% of cases, MRI showed more abnormalities than ultrasound (USG), in a further 21.8\%, MRI diagnosis was completely different, in 4.7\%, a pathology described on a USG was ruled out on MRI. The superiority of MRI over USG was $63.1 \%$.

Conclusions. MR-compatible incubator significantly increased the availability of MRI to newborns, especially to premature and unstable newborns. The integration of body coils into the INC increased the spectrum of examinations and made possible the scanning not only of the brain but also the body. Dedicated neonatal coils improved image quality and allowed more accurate diagnosis than the previously used adult coils. Immobilization of the babies in the INC by means of Velcro belts and head fixation inserts is better than in adult coils. The closed space of the INC isolates newborns to a greater extent from the negative influence of noise in the MR environment.

Key words: body imaging, brain imaging, magnetic resonance imaging, MR-compatible incubator, neonates 


\section{Introduction}

Since the time when we published our first experience with magnetic resonance imaging (MRI) in neonates with the use of a specialist incubator compatible with an MRI system (INC), ${ }^{1} 5$ more papers including the keywords "MRI", "neonates" and "MR compatible incubator" have been added to PubMed and 2 of these are ours. ${ }^{2,3} \mathrm{Al}$ though the first publication concerning INC dates back to $2003,{ }^{4}$ there are only 22 articles devoted to this subject in PubMed now (including 3 of ours) and almost all of them are focused on safety issues and brain imaging. ${ }^{4-7}$ Our INC, equipped with a head coil and a body coil, allows more than neuroimaging, and we have noticed the growing role of neonatal body MRI in everyday clinical practice. Our first reports concerned the first 27 and 47 newborns examined, respectively., ${ }^{1,3}$ Now we present our experience with brain and body MRI after having performed 555 of the procedures at the Institute of Mother and Child in Warszawa, Poland.

\section{Material and methods}

\section{Patients}

Over 4 years (2013-2017), we performed 555 MRI examinations (in 287 boys and 268 girls) in 530 neonates (275 boys, 255 girls), always after ultrasound (USG), performed by a radiologist. Institutional Bioethics Committee approval was acquired even though this is a retrospective study (decision No. 26/2017). In each case, written informed consent for clinical MRI was obtained from the patients' parents or legal guardians. In urgent cases, in the absence of parents/guardians, consent to the study was signed by a commission of 3 doctors. The examinations were always performed in the presence of an anesthesiologist.

\section{Equipment}

All neonates were examined using a GE Signa HDxT 1.5 T system (GE Healthcare, Chicago, USA) and MRcompatible incubator Nomag IC 1.5 by Lammers Medical Technology Co. (Lübeck, Germany), with temperature and humidity adjustment, equipped with non-magnetic gas cylinders with a capacity of $5 \mathrm{~L}$ of oxygen at a maximum pressure of 200 bar, with a non-magnetic gas cylinder pressure reducing valve with flow adjustment in the range of $0-5 \mathrm{~L} / \mathrm{min}$, respirator with a non-magnetic air pressure reducing valve, pulse oximeter measuring heart rate within the range of 20-300 bpm and partial oxygen pressure within the range of $1-100 \%$. From the point of view of imaging, the most important equipment were 3 MRI coils: An 8-channel, phased-array neonatal head coil and a 12-channel phased-array coil for the body, consisting of an 8-channel coil integrated in the INC bed and a separate 4-channel surface coil. The neonates were immobilized with Velcro belts and head fixation inserts. Separate neonatal noise attenuators were not used as the dual-wall structure of the INC lowers the noise level around the patient by approx. $12-15 \mathrm{~dB}$ and the head fixation inserts mentioned above provide further protection.

\section{Anesthesia}

To induce anesthesia, atropine at a dose of $0.01 \mathrm{mg} / \mathrm{kg}$ of body weight and thiopental at a dose of $4-7 \mathrm{mg} / \mathrm{kg}$ were used in all children. Four patients from the study group required respiratory support with a ventilator integrated with the INC, the others kept their own breath. In the freely breathing group, passive oxygen therapy was used in the INC. Vital signs, heart rate and oxygen saturation were monitored using a wireless oximeter adapted to work in a magnetic field. The medical gases came from non-magnetic cylinders. The babies did not require a catecholamine supply. During the study, due to a lack of infusion pumps adapted for operation in a magnetic field, the supply of intravenous fluids was stopped. The time without infusion did not exceed $30 \mathrm{~min}$.

\section{Image analysis}

Assessment of the quality of the MR examinations was performed on the basis of a visual inspection. The images were assessed in agreement by 2 radiologists $(\mathrm{MBF}$, 26 years of experience with MRI, 21 years of experience with neonatal MRI, and one of the remaining authors) in each case and final diagnoses were reached by consensus. The superiority of MRI over USG was defined as the MR visualization of elements and features that were not visualized by USG.

\section{Results}

\section{Characteristics of patients}

Most of the neonates were prematurely born: in 286/530 cases (54\%), they were born at a gestational age (GA) of $22+6-36+6$ weeks. For the whole study group, the mean age at birth was 32.5 gestational weeks (GW) (range 22-41 GW). Neonates were examined from the $1^{\text {st }}$ day of postnatal life to 153 days (mean: on the $37.7^{\text {th }}$ day of life), depending on clinical status and suspected pathology. Seventeen neonates $(3.2 \%)$ were scanned on the $1^{\text {st }}$ day of postnatal life and 124 neonates $(23.4 \%)$ within the $1^{\text {st }}$ week. On the day of the MRI, the body weight of the newborns ranged between $600 \mathrm{~g}$ and $5000 \mathrm{~g}$, with a mean of $3051 \mathrm{~g}$ (median $3040 \mathrm{~g})$. At the moment of the examination, 109 neonates (19.6\%) had body weight of less than $2500 \mathrm{~g}, 16$ (2.9\%) of less than $1500 \mathrm{~g}$ and $3(0.5 \%)$ of less than $1000 \mathrm{~g}$. 


\section{Characteristics of examinations}

Five-hundred eighteen examinations (518/555 = 93.5\%) were performed under pharmacological sedation, 37 (6.5\%) in physiological sleep. There were no anesthetic complications in our material.

In the analyzed material there were 533 brain examinations, 5 studies of orbits, 21 of the vertebral column, spinal canal and cord, 18 of the head and neck, 6 of the thorax, 33 of the abdomen and pelvis, and 2 of joints. In 31 neonates we scanned 2 body regions, in 3 babies 3 regions and in 2 babies 4 regions, at one time. Therefore, 555 procedures totaled 598 examined regions of the body. Twenty-four babies had an initial examination and a follow-up study and 1 was scanned 3 times. The proportion of brain MRIs to the scans of other body regions was $533: 85$, i.e., $86 \%$ vs $14 \%$.

The quality of MR examinations was found satisfactory in all but 4 cases (99.3\%). In 1 of these examinations, the neonate was thought to be in natural sleep but started crying, but the quality was sufficient to confirm the continuity of the spinal cord which was questioned by the parents. In 2 cases anesthesia was very difficult and the studies were stopped, but the completed sequences revealed no evident brain pathology. In the $4^{\text {th }}$ case, MRI of the brachial plexi was requested and it was impossible to assess the particular nerve roots forming the plexi due to insufficient spatial resolution.

\section{Result of magnetic resonance imaging versus ultrasonography}

In 203 cases $(203 / 555=36.6 \%)$ the MRI showed more abnormalities than the USG and in a further 121 cases $(121 / 555=21.8 \%)$ the diagnosis after MRI was completely different than after USG. In 26 cases $(26 / 555=4.7 \%)$ the pathology described on the USG was ruled out on MRI. The superiority of MRI over USG in the visualization of pathological lesions and normal structures was found in $63.1 \%$ of cases in the collected material in neuroimaging and body imaging.

\section{Discussion}

\section{General remarks}

Ultrasonography is and will remain the first-line imaging method in neonates. However, it has its well-known limitations and additionally, some parts of the brain, e.g., the sella, and of the body, e.g., the chest, do not lend themselves to USG examination and are most appropriately examined using MRI. Computed tomography $(\mathrm{CT})$ is another option but according to the ALARA rule (as low as reasonably achievable), the radiologist should establish a diagnosis with the use of a radiation dose that is as low as possible and no dose of ionizing radiation is the best solution. Moreover, the tissue resolution of $\mathrm{CT}$ is much lower than that of MRI so the routine use of $\mathrm{CT}$ in the youngest group of patients is not justified. Therefore, MRI, by definition, has higher diagnostic usefulness, not only in this age group.

\section{The added value of MR-compatible incubator}

Some national societies have published recommendations on the use of MRI in neonates, preterm in particular. ${ }^{8,9}$ Safety issues such as transport of the neonates to MRI units, maintaining the temperature and monitoring vital functions have limited performing MRI so far, especially in premature infants. The equipment of the MR-compatible incubator, making it possible to control the temperature and humidity and to maintain and monitor vital parameters, ensures safe conditions for the babies. Therefore, even preterm and unstable neonates can be scanned safely now. Skeptics argue that the advantages of INC are unclear: it does not obviate the need for anesthesia or sedation and it even obscures the anesthesiologist's view of the patient; neonatal coils are available without the INC; transport can be safely accomplished in a standard neonatal incubator after placing the patient onto the MRI table with appropriate hearing protection; intravenous fluids can be administered with the pumps kept outside of the MRI chamber and tubes running through a hole in the wall or with MR-compatible pumps. However, before the era of INC, neonatologists would not entrust their most preterm and unstable neonates to radiologists, at least because of the impossibility to maintain proper temperature, and also because the whole procedure is complex. Introduction of INC to the clinical practice allowed examination of even the smallest neonates with extremely low body weights - our smallest neonate, weighing $600 \mathrm{~g}$, is still the smallest neonate examined to date, as shown in the available literature, ${ }^{1}$ although followed very closely by a 620 -gram baby reported in the newest paper devoted to this subject that was published last year. ${ }^{10}$ In our material, as many as more than half of the examined neonates were preterm (54\%) and almost $1 / 4$ (23\%) weighed less than $2500 \mathrm{~g}$ - equivalent of low birth weight (LBW) - on the day of MRI. Seventeen neonates (3.2\%) were scanned on the $1^{\text {st }}$ day of postnatal life and $124(23.4 \%)$ within the $1^{\text {st }}$ week. Before introduction of INC, MRI was performed on such newborns only in exceptional situations.

Our material constitutes the largest cohort of neonates examined in INC to date. Cho et al. reported 154 newborns over the course of more than 4 years. ${ }^{10}$ Earlier reports are based on much smaller groups of neonates (from 13 to 129). ${ }^{6,7}$ Our group of 383 neonates with $400 \mathrm{MR}$ examinations performed was collected in 3 years (2013-2016), which reflects the need for these studies in specialized tertiary referral centers.

\section{Anesthesia}

In the vast majority of MRI examinations in INC - 93.5\% - pharmacological sedation was used. In $6.5 \%$ of cases, after feeding $30 \mathrm{~min}$ before scanning, we achieved physiological 
sleep peaceful enough to be able to perform the procedure during it. Despite comfortable conditions inside the INC (optimal temperature and humidity, insulation from noise), in most newborns physiological motion artifacts occur during sleep hindering the precise evaluation of the images. In children studied in natural sleep, attempts have been made to use pacifiers to calm them down, but motion artifacts associated with suction made us abandon this method. Another argument against placing the just-fed neonate in an INC chamber is the risk of regurgitation and aspiration of food into the airways. Premature neonates are subject to this risk to the greatest extent. During scanning, newborns must be in a horizontal position, the upper body cannot be lifted to prevent aspiration. One should also keep in mind that an anesthesiologist does not have visual supervision of a baby in INC. His or her assessment is based on the recordings of the monitoring equipment, which gives results with a delay of tens of seconds, which may have impact on the effect of rescue operations in case of aspiration of food into the airways. Taking into account the above considerations, the authors believe that only neonates in good condition may be subjected to MRI in physiological sleep: neonates with no history of episodes of aspiration pneumonia and those in whom MRI is carried out as part of planned prospective control programs, where the inevitable motion artefacts do not have significant impact on the precision of image evaluation. It should also be remembered that conversion of the procedures towards anesthesia is possible only after $6 \mathrm{~h}$ after feeding in fed infants in case of failure to obtain physiological sleep allowing the execution of MRI.

At our center, the vast majority of children undergoing MRI in INC are premature neonates with congenital malformations, CNS hemorrhages or after severe perinatal hypoxia. In these babies, the results of MRI have great therapeutic and prognostic implications, which is why this procedure - in order to obtain optimal image quality - is performed under general anesthesia although the use of sedation is discussed in the literature and some authors stress that the sedative drugs may have a negative influence on the immature brain of extremely premature babies. ${ }^{11-13}$ However, sedation is used worldwide and, e.g., Cho et al. report that the oral sedative agent chloral hydrate was used in their babies when possible, and in other cases midazolam or ketamine hydrochloric acid were administered intravenously. ${ }^{10}$

There were no anesthetic complications in the examined newborns in our material.

\section{Brain magnetic resonance imaging}

The study of the brain is now an important challenge in neonatology and the development of imaging techniques and - above all - of MRI offers great opportunities for assessment and analysis of brain morphology in vivo. At the same time, a lot of questions are being posed about the place of MRI as a tool for assessment and prognosis of the development of newborns, both those born at term and those extremely premature. In the USA, at least since 2004, it is recommended to perform MRI at term equivalent in preterm babies due to its prognostic value and to perform MRI if any neurological symptoms are present, as diffusion-weighted sequence (DWI) in particular is capable of reliable assessment of CNS injury. ${ }^{14}$ The date, 2004, coincides with the time of the introduction of incubator into clinical practice. ${ }^{4}$

The brain can be injured at any stage of its development, and the damage may be congenital (developmental abnormality) or acquired (e.g., hypoxic-ischemic or hemorrhagic). There is no doubt that transfontanelle USG is and will remain the primary diagnostic method - non-invasive, readily available and cheap. But it has limitations, and at present MRI is the most accurate method of brain imaging. Thanks to visibility of all the structures, including basal ganglia, internal capsule, brainstem and cerebellum, and the possibility to measure the volume of these structures (e.g., cerebellum), MRI provides additional information about which drug treatment or rehabilitation can be implemented and facilitates the prognosis for the babies' further development.

Neurologists emphasize that each stage of brain development is very important, but the formation and selection of synapses and myelination seem to be the most important for the brain. This type of information can only be obtained using MRI, but images must be of very high quality and newborns subjected to MRI may be in various clinical conditions: from stable and apparently healthy to unstable.

Among neonates born at term, the study is performed most often in the first days of life and mainly in 2 clinical situations: birth defects and after mild to severe perinatal hypoxia, a few days after therapeutic hypothermia. Sometimes these newborns require respiratory support (from $\mathrm{nCPAP}$ to endotracheal intubation and mechanical ventilation) and inotropic agents (catechol amines).

In the group of premature neonates, so far MRI has been performed only in exceptional situations in the first days of postnatal life, when their clinical condition is very unstable, because at that time USG is the primary diagnostic tool. Magnetic resonance imaging is performed more often between 36 and 40 weeks of postconceptional age as it brings additional information in relation to USG that may help predict prognosis for the neonates' further development. ${ }^{15}$

Our experience shows that MRI in INC is feasible in preterm newborns even on the first days of postnatal life (Fig. 1). This observation is in agreement with those of other authors who stated that the interval between birth and MRI was significantly shorter after the introduction of INC, i.e., $54.3 \pm 2.6$ days vs $70.5 \pm 4.4$ days without INC. ${ }^{10}$ Our results show even earlier availability of MRI in INC as compared to that of Cho et al., with a median of 18.5 days and mean of 37.7. When performed at term equivalent, 


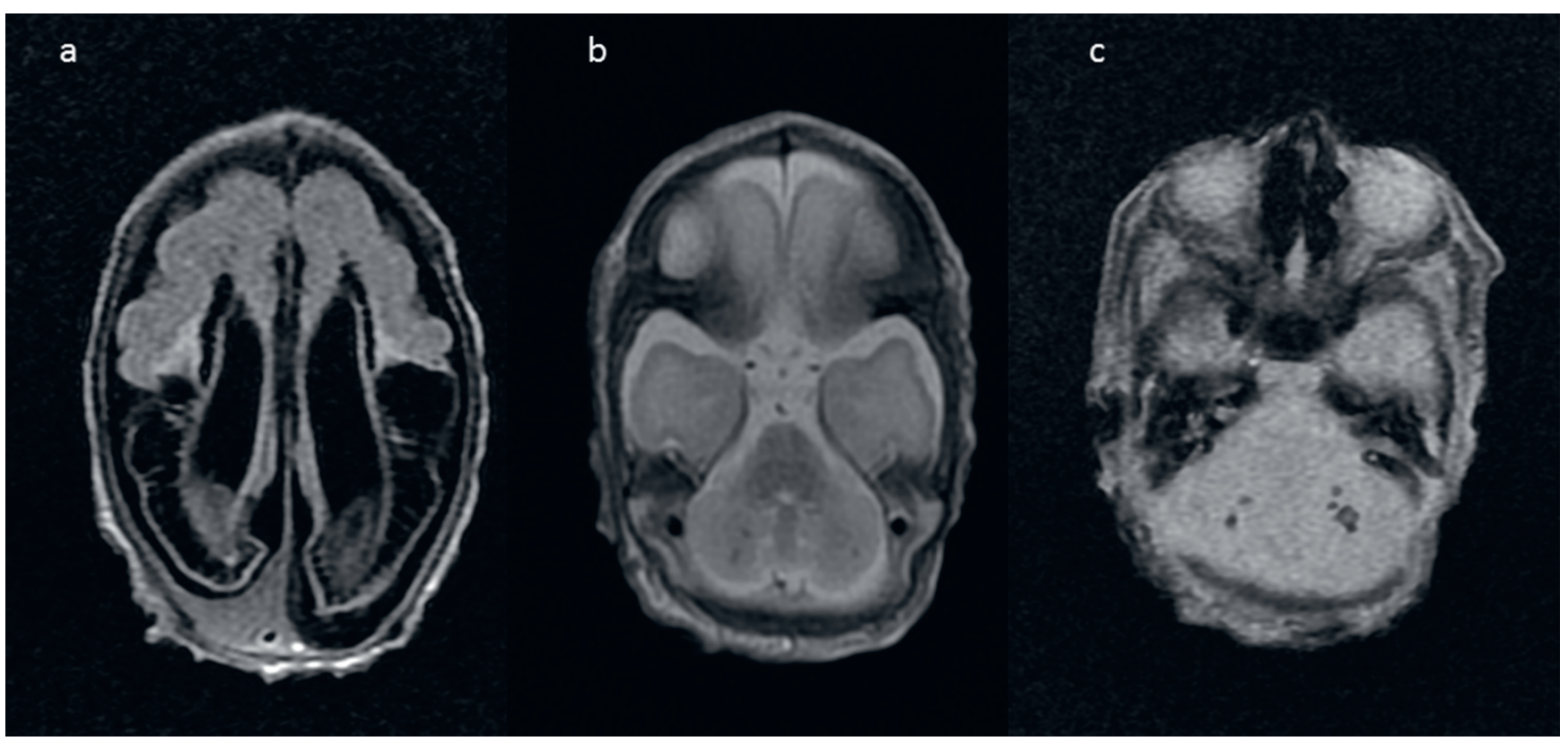

Fig. 1. Male preterm neonate born at $26 \mathrm{GW}$, examined at $29 \mathrm{GW}$. (a) FLAIR shows relatively small cavities in the frontal lobes and extensive encephalomalacia in the posterior parts of the brain. Pericerebral hematoma on the right. Axial FSE/T2-weighted (b) and GRE/T2*-weighted (c) images show bilateral cerebellar hemorrhages

MRI allows assessing brain injury in neonates with known pathology and reveal unexpected findings in neurologically and otherwise healthy ones. One example is a baby born at $27 \mathrm{GW}$ and examined at 38, before discharge, who turned out to have diffuse polymicrogyria involving almost the entire left cerebral hemisphere. Abnormal gyration is extremely difficult to be revealed sonographically. Magnetic resonance imaging is necessary in these cases and such a finding carries significant implications for the future. The dedicated neonatal coils in the INC provide better signal-to-noise ratio and smaller fields of view and these factors allow better visualization of small details. ${ }^{5,6,10}$ The percentage of cerebellar injury detected with MRI is incomparably greater than that diagnosed with USG. As in case of neuronal migration abnormalities, the damage to the cerebellum is often difficult to visualize and evaluate on USG and, in these cases, MRI is also a method of choice. Although mastoid fontanelle USG enables a better view of the posterior fossa, MRI shows more abnormalities and enables assessment of the real state of the cerebellum and brain stem in which the incidence of defects and damage turned out to be greater than expected. ${ }^{16,17}$ In a recent paper by Steggerda et al., USG allowed to detect abnormalities in the posterior fossa in $41 \%$ of infants (in the vast majority these abnormalities were only seen on mastoid fontanelle views) while MRI revealed them in $66 \% .{ }^{18}$ In our material, in 71 cases $(71 / 533=13.3 \%$ of brain scans $)$ the sonographic picture of the cerebellum was normal while MRI revealed cerebellar abnormalities (cases with hemorrhage only in pericerebellar space that was not visualized by USG were not counted) (Fig. 2A,B). Also, in the case of known pathology mentioned at the beginning of this paragraph, MRI can show its consequences which are occult to USG.
Examples are pre-Wallerian (and later Wallerian) degeneration or crossed cerebellar diaschisis, which carry significant clinical consequences. ${ }^{19,20}$ (Fig. 3A,B).

\section{Body imaging in MR-compatible incubator}

In these very few papers that have been published so far (very few despite the fact that INC was introduced to clinical practice at least 15 years ago), only neuroimaging was described. Only Blüml et al. mentioned 2 cardiac and 2 pelvic MR examinations in their paper, but it is mainly a technical and safety-concerning publication. By the way, Blüml et al. found that the signal-to-noise ratio (SNR) of images acquired with INC had been 2.3 times greater than the SNR of images acquired with standard "adult" equipment at MRI of age-matched babies. ${ }^{6}$ These authors used the same incubator and the same MRI system as in our case. O'Regan et al., using another vendor's $1.5 \mathrm{~T}$ scanner and the same INC, achieved a 3-fold increase of SNR in brain imaging. ${ }^{5}$ Our experience shows that neurological indications are still the most frequent in neonatal MRI (in our study, brain scans constituted the majority of cases $-86 \%$ ) but neurological indications include also spinal ones, and imaging of the vertebral column, spinal canal and cord is now possible and scheduled. Our material contains 21 cases of such examinations with diagnoses of myelomeningocele, diastematomyelia, tethered cord, or exclusion of suspected pathology (Fig. 4A,B). Although USG of the spinal canal and cord is a reliable method in newborns, and in patients with normal findings no further imaging is necessary in most cases, (neuro) surgeons usually request MRI before surgical intervention in the case of spinal malformations. The examination 


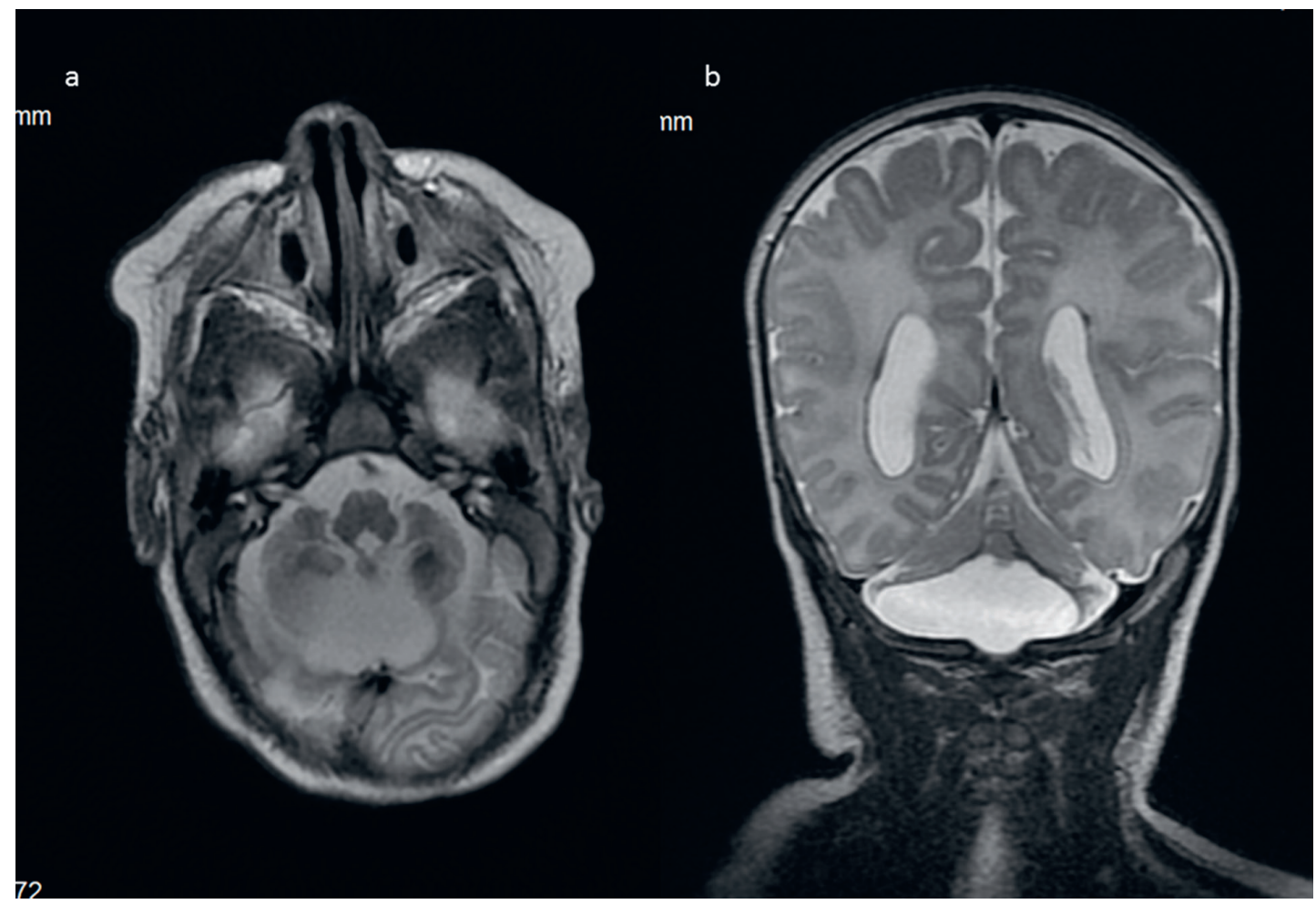

Fig. 2. Female second twin born at $\mathrm{GA}$ of 25 weeks, examined at 42. FSE/T2-weighted images in axial (a) and coronal projection (b). Posthemorrhagic destruction of both cerebellar hemispheres with hypointense deposits of hemosiderin and hyperintense cystic space between and below the hemispheres

is also performed for medical-legal reasons, e.g., when spinal cord injury is suspected due to traumatic birth. In our material, in 1 case this was the reason to perform spinal MRI when the depressed parents of a sick baby accused obstetricians of having injured the spinal cord secondarily to traction forces during delivery. The examination showed a normal spinal cord without evidence of any lesions.

What was shown in our study is that MR body imaging has started to play a growing role in neonatal care thanks to the development of body coils. This equipment consists of an 8-channel coil integrated in INC and a 4-channel surface coil as mentioned above. They enable scanning of not only the spine but also the neck, thorax, abdomen, and pelvis.

What we find significant is that neonatologists, surgeons and oncologists have started to refer neonates to non-CNS MRI thanks to the fact that we have body coils and to the safety of MRI in INC. There is no doubt that USG still is and will be the first line imaging modality, but whenever it is insufficient, MRI in INC is the most valuable and safest non-invasive tool that we have to answer clinical questions which cannot be answered by USG, and it is of utmost importance in neonates that must be operated on. In our material, we dealt with a female neonate whose mother did not see the obstetrician during pregnancy and did not receive prenatal USG. The baby was diagnosed with persistent cloaca after birth and surgical repair was scheduled, but MRI turned out to be necessary to confirm a sonographic diagnosis of bilateral renal agenesis and agenesis of the bladder before the decision to give up treatment.

We have already described a case in which the association of abdominal cyst with the biliary ducts was excluded. ${ }^{3}$
In another baby, this relationship was found on MRI and MR-cholangiography, and a common bile duct cyst was diagnosed implying referral of the newborn to another tertiary reference center that deals with biliary malformations. There is also a case of gallbladder duplication in our material.

In the case of lymphangioma, its extent frequently cannot be assessed by means of USG, e.g., if it penetrates to the retropharyngeal space or to the mediastinum, as it was in one of our babies. Magnetic resonance examinations of the chest belong to the least frequent in the analyzed material, which is quite obvious knowing the limited (at least so far) value of this method in this part of the human body. In another case, we easily visualized the lesion, its smooth borders and fluid contents, but the question whether this was a bronchogenic cyst or a cystic teratoma remained unsolved.

In another case, the results of USG were equivocal, indicating a complex ovarian cyst or presacral teratoma. Different surgical approaches required unequivocal diagnosis and MRI gave it, clearly showing sacrococcygeal teratoma type IV according to Altman.

Inborn tumors are a challenge for all involved specialists, especially when unclear at presentation. Magnetic resonance imaging was also performed at our center after discrepant orthopedic and sonographic evaluations suggesting a congenital anomaly of the left hip and unspecified mass, respectively. Magnetic resonance imaging revealed a giant tumor of the left buttock and thigh extending to the pelvis, inseparable from the rectum and vagina, with enlarged lymph nodes in both groins, around the iliac vessels and the aorta up to the level of the diaphragm. After 


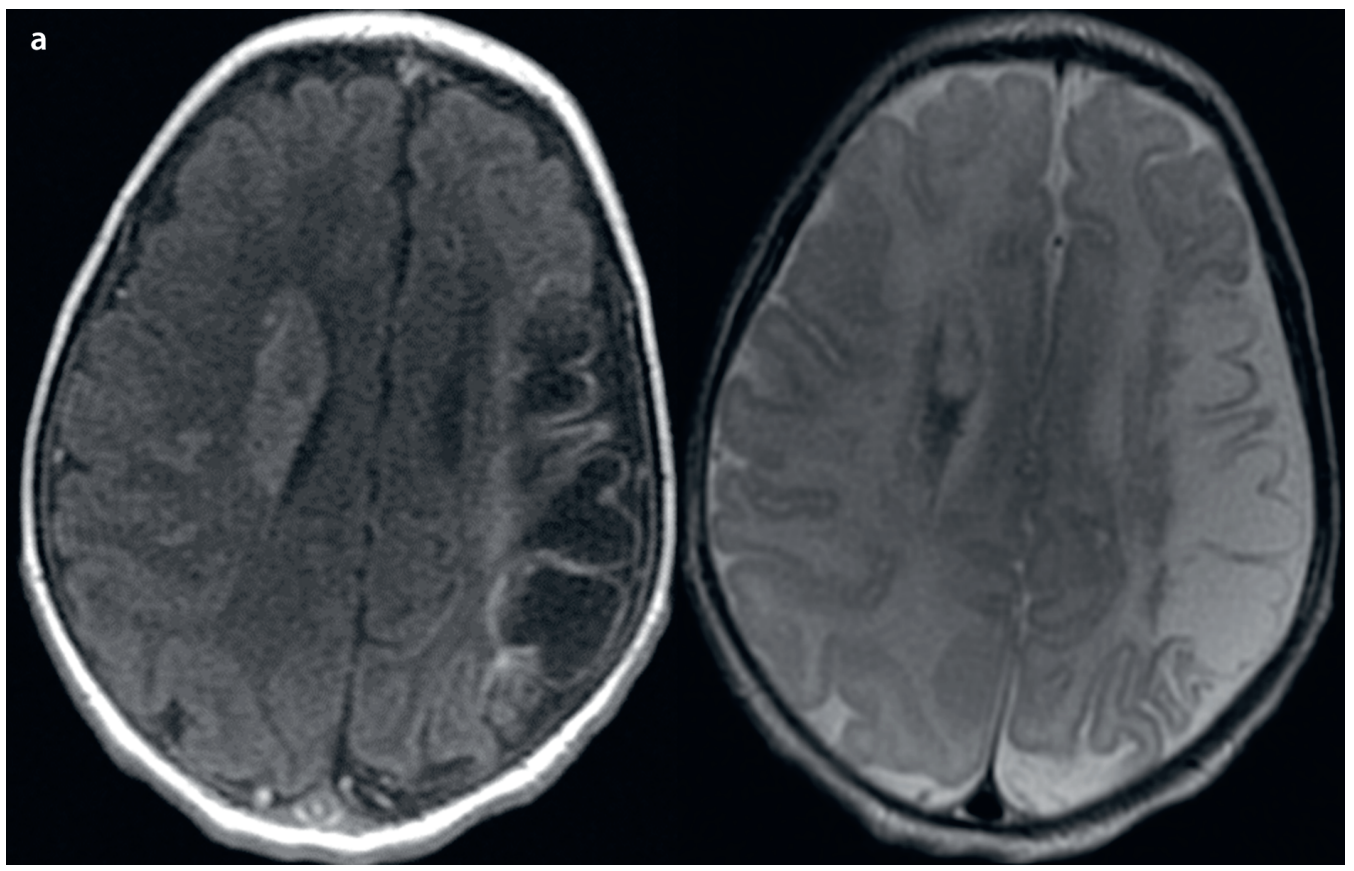

Fig. 3. Female neonate born at the age of $40 \mathrm{GW}$ and examined at the age of 8 days.

a) SE/T1- and corresponding FSE/T2-weighted images in axial plane show the area of "old" stroke in the region supplied by the left middle cerebral artery, which must have occurred prenatally, and the smaller area of new insult in the deep structures of the right cerebral hemisphere.

b) DWl images (upper row) and corresponding ADC maps (lower row) show diffusion restriction in the area of "new" stroke on the right and simultaneously occurring (pre-)Wallerian degeneration going down to the brain stem along the corticospinal tracts. Note the decreased volume of the left cerebral peduncle, which represents earlier Wallerian degeneration on the left, due to the earlier stroke

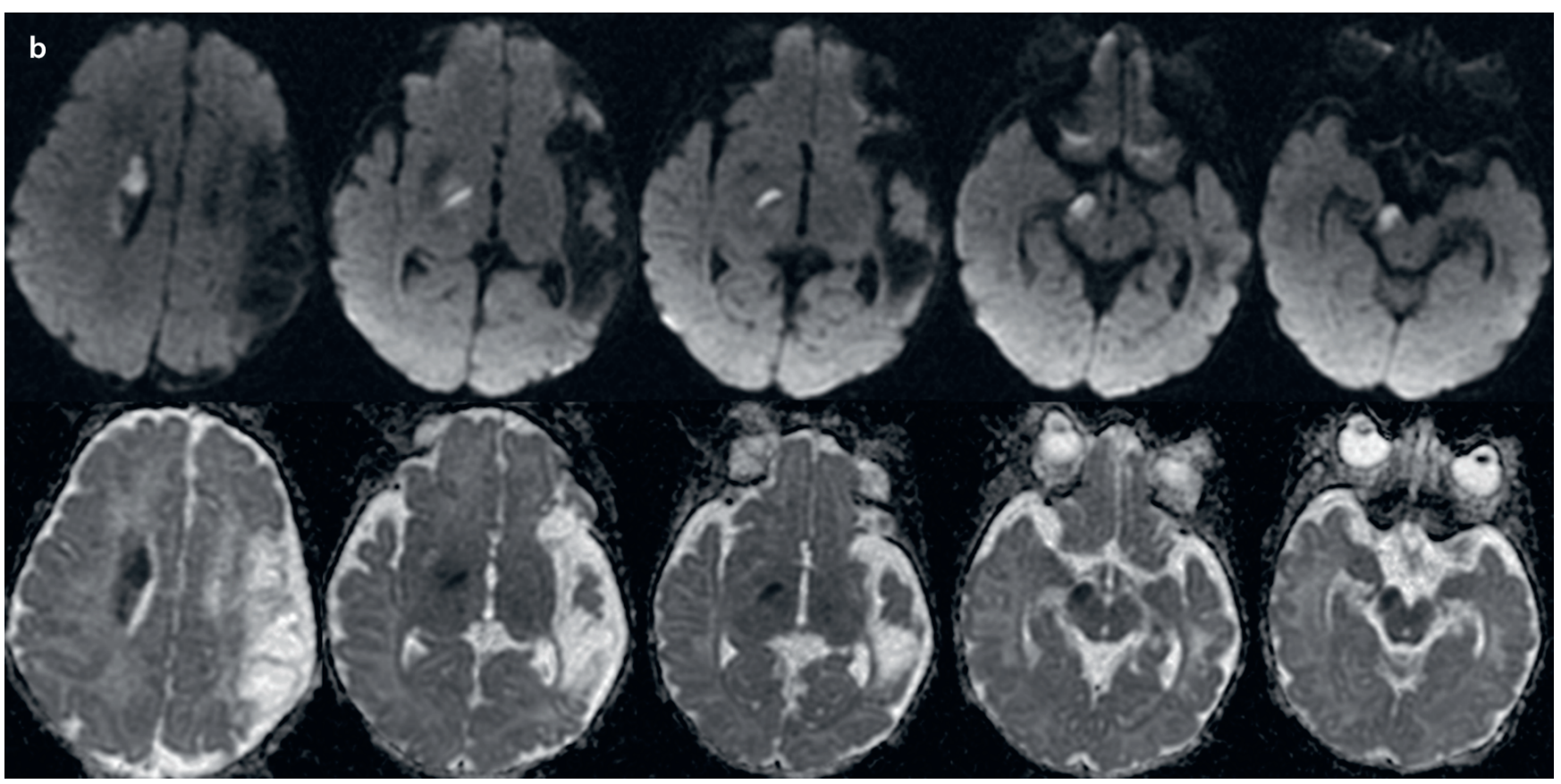

biopsy and numerous pathologic consultations, a diagnosis of infantile myofibroma was established.

As stated before, literature concerning neonatal MRI in INC is very scant and there are no descriptions of body imaging. In this aspect, our observations are the first in world literature. In our material, MRI body scans constituted $14 \%$ and, to the best of our knowledge, this is the first report on body scanning in INC in a large cohort of neonates. Our results show that body imaging in INC is feasible and more and more frequently required, not only to establish diagnosis but also to follow up the patient after surgery. One example is a follow-up examination after resection of huge facial hemangiopericytoma with no residual disease. In our material, we have 2 cases of serial follow-up studies in INC in preterm neonates who were examined due to unclear sonographic appearance after surgery for sacrococcygeal teratoma, with a diagnosis of pelvic abscess in 1 case (Fig. 5) and pelvic hematoma in the other, and subsequently - the regression of lesions. Residual or recurrent tumors were thus ruled out.

\section{Final remarks}

Altogether, in $36.6 \%$ of our material, MRI showed more abnormalities than USG and in a further $21.8 \%$, MRI changed the diagnosis. In $4.7 \%$ of cases, pathological lesions noted on USG were ruled out on MRI. The superiority of MRI over USG was $63.1 \%$ in the collected material in neuroimaging 


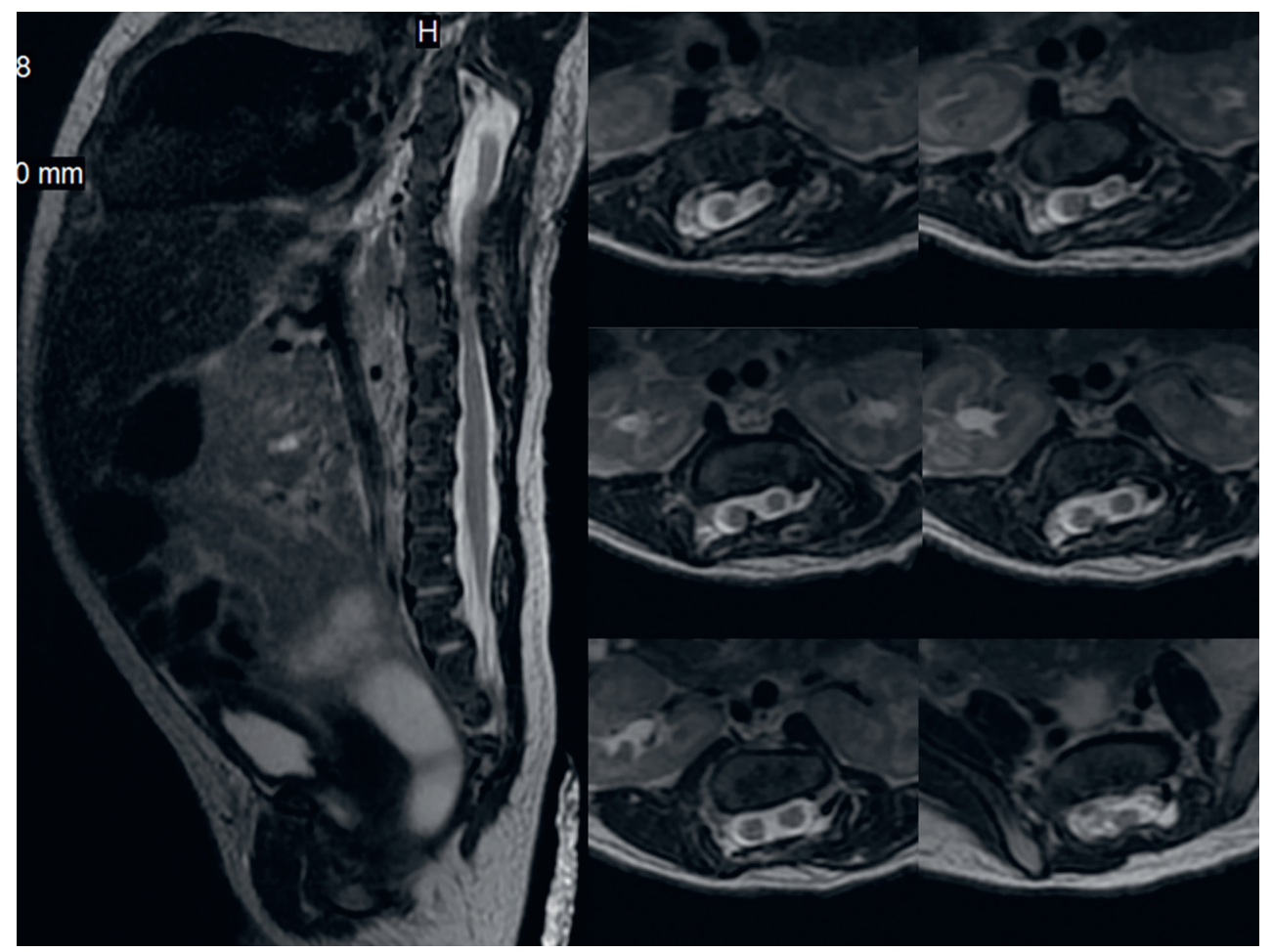

Fig. 4. Female term neonate examined at the age of 7 days.

FSE/T2-weighted images in sagittal A) and axial B) projection. Vertebral anomalies, tethered cord A) and diastematomyelia B) are observed
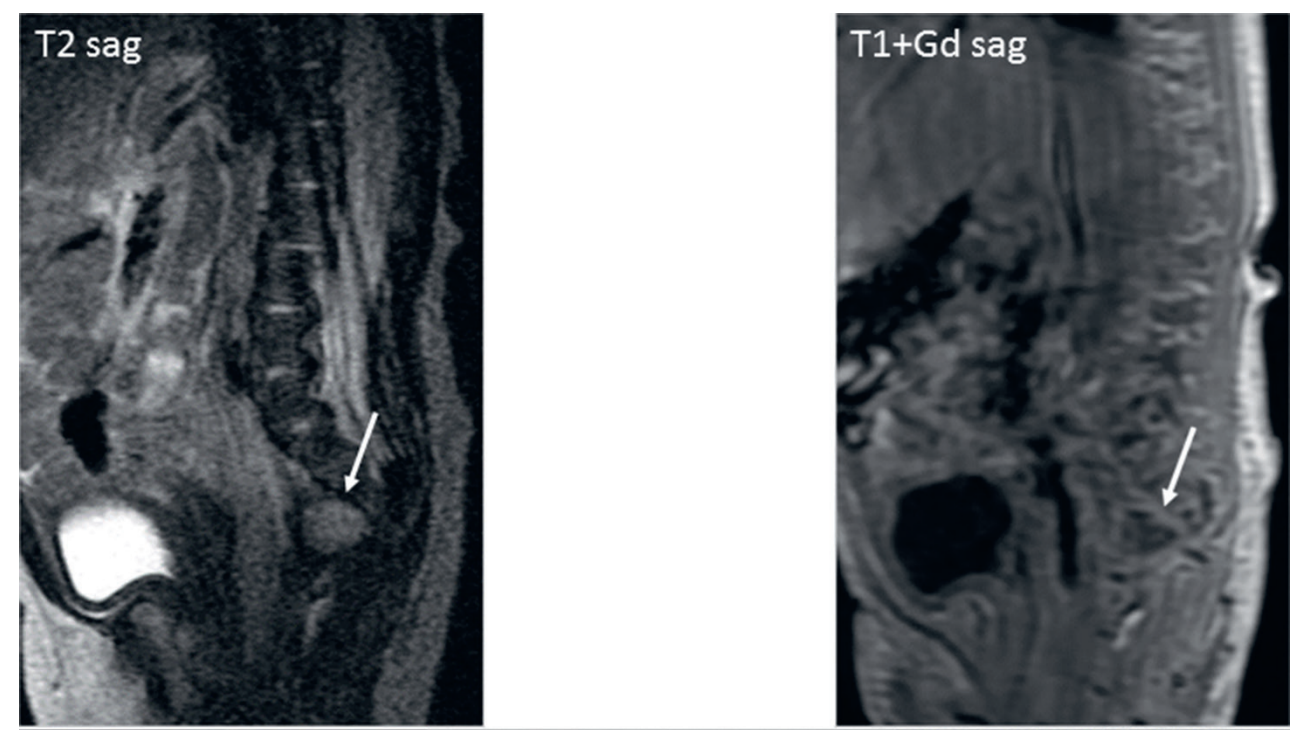

Fig. 5. Follow-up study still

in the neonatal period after surgery for sacrococcygeal teratoma due to inconclusive sonographic findings. A lesion with enhancing wall, filled with thick fluid showing diffusion restriction was typical of abscess (arrows)

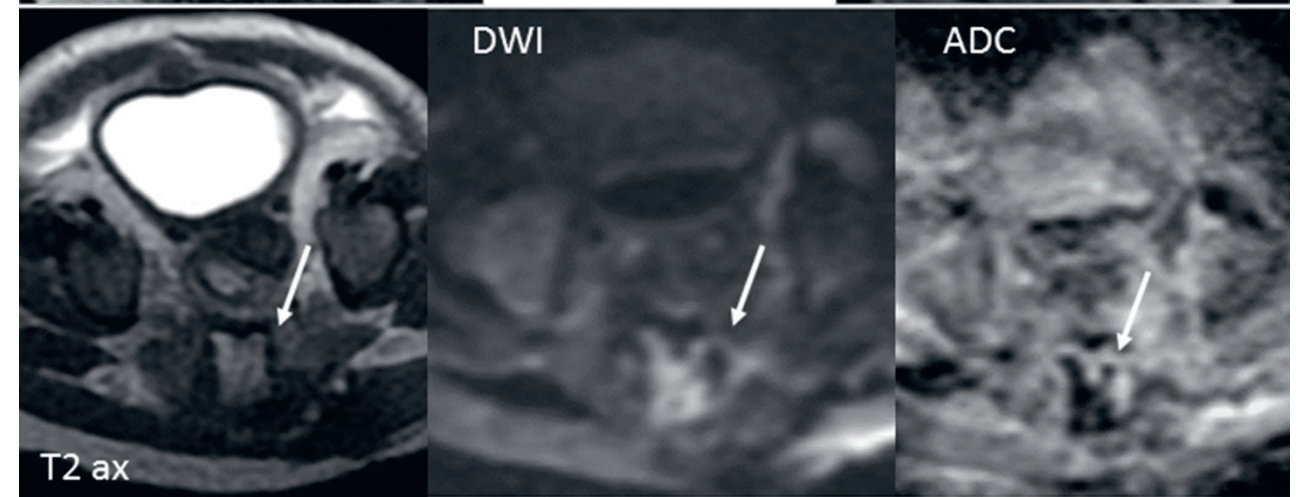

at the moment. A good example is the abovementioned case

and body imaging. Obviously, picking up more abnormalities (i.e., higher sensitivity) with MRI does not necessarily equate to a change of the care that the patient receives of diffuse polymicrogyria detected at the asymptomatic moment of the baby's life. However, even if such a finding 
causes increased parental anxiety, it is crucial for the prognosis and spares future examination when seizures appear. So the significance of MRI findings is not only related to the change of current therapeutic procedures, but also to the outcome and implications for the child's future life. This increased sensitivity of MRI makes possible progress in medical knowledge as well, as in the case of the very littledescribed issue of pre-Wallerian degeneration in the corticospinal tracts and in the corpus callosum after brain insult. ${ }^{20}$ This increased sensitivity of MRI; however, is not always equivalent to finding clinically meaningful information. The classic example of this is diffuse excessive high signal intensity (DEHSI) of the white matter seen on neonatal MRI and invisible on USG, which is a highly qualitative finding and does not appear to have clear clinical significance. ${ }^{21}$ In our material we did not count DEHSI as an element of MRI superiority over USG.

Although we have been trying for years to convince clinicians that prenatal MRI can be the basis of treatment after birth, it still happens that they wish to have postnatal confirmation of radiological prenatal findings, which is relatively easily available with INC. In our study, we have shown that in most such cases, there was no added value of postnatal MRI as compared to prenatal. However, MRI in INC allowed a visualization of small details that could not have been noticed on prenatal MRI or required contrast medium administration to be noticed. ${ }^{2}$

\section{Limitations of MR-compatible incubator}

The size of an extracorporeal lesion is one of the limitations of the use of INC. Besides the presented material, we had 2 newborns who did not fit into the space provided in the head coil: one with suboccipital encephalocele of large dimensions and another one with a tongue tumor which turned out to be part of blue rubber bleb nevus syndrome. The coil could not be closed around the neonate's heads and we had to give up INC. Another limitation is spatial resolution of the body coil. For instance, brachial plexus injury can be visualized if edema is seen within it. However, in the absence of edema, it is practically impossible to follow the particular nerve roots leaving the spinal canal in the cervical and thoracic region and forming the plexus. The lesions in the extremities limit the use of INC - in the absence of small local coils we had to give up INC in a newborn with congenital rhabdomyosarcoma of the forearm. There is still room for technical progress, but progress has already been made by introducing body coils to the INC.

\section{Conclusions}

In conclusion, the MR-compatible incubator constitutes a significant advancement in neonatal diagnostics. It has significantly increased the availability of MRI to newborns, especially those who are premature or otherwise unstable.
Integration of body coils into INC has increased the spectrum of possible examinations and made possible scanning not only of the brain but also of the body. Dedicated neonatal coils have improved image quality and allowed more accurate diagnosis than the adult coils used previously. The immobilization of the neonate in INC with the use of Velcro belts and head fixation inserts are better than in adult coils. The closed space of INC isolates newborns to a much greater extent than before from the negative influence of noise in the MR environment.

\section{References}

1. Bekiesińska-Figatowska M, Szkudlińska-Pawlak S, Romaniuk-Doroszewska A, et al. First experience with neonatal examinations with the use of MR-compatible incubator. Pol J Radiol. 2014;79:268-274.

2. Bekiesińska-Figatowska M, Romaniuk-Doroszewska A, Duczkowska A, Duczkowski M, Iwanowska B, Szkudlińska-Pawlak S. Fetal MRI versus postnatal imaging in the MR-compatible incubator. Radiol Med. 2016;121(9):719-728.

3. Bekiesińska-Figatowska M, Helwich E, Rutkowska M, Stankiewicz J, Terczyńska I. Magnetic resonance imaging of neonates in the magnetic resonance compatible incubator. Arch Med Sci. 2016;12(5): 1064-1070.

4. Erberich SG, Friedlich P, Seri I, Nelson MD Jr, BlümI S. Functional MRI in neonates using neonatal head coil and MR compatible incubator. Neuroimage. 2003;20(2):683-692.

5. O'Regan K, Filan P, Pandit N, Maher M, Fanning N. Image quality associated with the use of an MR-compatible incubator in neonatal neuroimaging. Br J Radiol. 2012;85(1012):363-367.

6. BlümI S, Friedlich P, Erberich S, Wood JC, Seri I, Nelson MD Jr. MR imaging of newborns by using an MR-compatible incubator with integrated radiofrequency coils: Initial experience. Radiology. 2004;231(2): 594-601.

7. Rona Z, Klebermass K, Cardona F, et al. Comparison of neonatal MRI examinations with and without an MR-compatible incubator: Advantages in examination feasibility and clinical decision-making. Eur J Paediatr Neurol. 2010;14(5):410-417.

8. Helwich E, Bekiesińska-Figatowska M, Bokiniec R. Standard badań obrazowych oun noworodka. In: Standardy opieki medycznej nad noworodkiem w Polsce. Zalecenia Polskiego Towarzystwa Neonatologicznego. Wydawnictwo Media-Press Sp.z o.o., Warszawa 2015, 150-157.

9. Fetal and Neonatal Brain Magnetic Resonance Imaging: Clinical Indications, Acquisitions and Reporting. A Framework for Practice. British Association of Perinatal Medicine (BAPM), February 2016. www. bapm.org

10. Cho HH, Kim IO, Cheon JE, Choi YH, Lee SM, Kim WS. Changes in brain magnetic resonance imaging patterns for preterm infants after introduction of a magnetic resonance-compatible incubator coil system: 5-year experience at a single institution. Eur J Radiol. 2016;85(9): 1564-1568.

11. Loepke AW, McGowan FX Jr, Soriano SG. CON: The toxic effects of anesthetics in the developing brain: The clinical perspective. Anesth Analg. 2008;106(6):1664-1669.

12. Jevtovic-Todorovic V. Developing brain and general anesthesia - is there a cause for concern? F1000 Med Rep. 2010;8(2):68.

13. Wilder RT. Is there any relationship between long-term behavior disturbance and early exposure to anesthesia? Curr Opin Anaesthesiol. 2010;23(3):332-336.

14. Neil JJ, Inder TE. Imaging perinatal brain injury in premature infants. Semin Perinatol. 2004;28(6):433-443.

15. Zupan-Simunek V, Rutkowska M, Bekiesińska-Figatowska M. Predictive value of magnetic resonance imaging (MRI) in cases of acquired brain injury in neonates. Med Wieku Rozwoj. 2011;15(3 Pt 2):385-393.

16. Biran V, Bodiou AM, Zana E, et al. Cerebellar injury in premature infants less than 30 weeks of gestation. Arch Pediatr 2011;18(3):261-266.

17. Bekiesińska-Figatowska M, Jurkiewicz E, Szkudlińska-Pawlak S, Malczyk K, Nowak K. Rhombencephalosynapsis - isolated anomaly or complex malformation? Pol J Radiol. 2012;77(3):35-38. 
18. Steggerda SJ, de Bruïne FT, Smits-Wintjens VE, Verbon P, Walther FJ, van Wezel-Meijler G. Posterior fossa abnormalities in high-risk term infants: Comparison of ultrasound and MRI. Eur Radiol 2015;25(9): 2575-2583.

19. Bekiesińska-Figatowska M, Duczkowski M, Madzik J, Uliasz M Zawadka A, Baszczeska J. Diffusion-weighted imaging of the early phase of wallerian degeneration. A report of two pediatric cases and literature review. Neuroradiol J. 2012;25(6):657-664.
20. Bekiesińska-Figatowska M, Duczkowska A, Szkudlińska-Pawlak S, et al. Diffusion restriction in the corticospinal tracts and the corpus callosum in neonates after cerebral insult. Brain Dev. 2017;39(3): 203-210.

21. Broström L, Bolk J, Padilla N, et al. Clinical implications of diffuse excessive high signal intensity (DEHSI) on neonatal MRI in school age children born extremely preterm. PLoS One. 2016;11(2):e0149578. 